\title{
LINEAR LAYER AND GENERALIZED REGRESSION COMPUTATIONAL INTELLIGENCE MODELS FOR PREDICTING SHELF LIFE OF PROCESSED CHEESE
}

\author{
Sumit Goyal, Senior Research Fellow \\ Gyanendra Kumar Goyal, Emeritus Scientist
}

National Dairy Research Institute, Karnal, India

E-mail: thesumitgoyal@gmail.com,gkg5878@yahoo.com

Received March 16, 2012

\begin{abstract}
This paper highlights the significance of computational intelligence models for predicting shelf life of processed cheese stored at 7-8 $\mathrm{C}$. Linear Layer and Generalized Regression models were developed with input parameters: Soluble nitrogen, $p H$, Standard plate count, Yeast \& mould count, Spores, and sensory score as output parameter. Mean Square Error, Root Mean Square Error, Coefficient of Determination and Nash - Sutcliffo Coefficient were used in order to compare the prediction ability of the models. The study revealed that Generalized Regression computational intelligence models are quite effective in predicting the shelf life of processed cheese stored at $7-8^{\circ} \mathrm{C}$.
\end{abstract}

\section{KEY WORDS}

Artificial Intelligence; Artificial Neural Network; Linear Layer; Generalized Regression; Processed Cheese; Shelf Life Prediction.

Linear Layer and Generalized Regression Artificial Neural Network (ANN) models were developed for predicting shelf life of processed cheese stored at $7-8^{\circ} \mathrm{C}$.

Artificial Neural Networks, also known as "artificial neural nets" or "neural nets", are a computational tool modeled on the interconnection of the neuron in the nervous systems of the human brain and that of other organisms. The term "neural net" refers to both the biological and artificial variants, although typically the term is used to refer to artificial systems only. Mathematically, neural nets are nonlinear. Each layer represents a non-linear combination of non-linear functions from the previous layer. Each neuron is a multiple-input, multiple-output (MIMO) system that receives signals from the inputs, produces a resultant signal, and transmits that signal to all outputs. Practically, neurons in an ANN are arranged into layers. The first layer that interacts with the environment to receive input is known as the input layer. The final layer that interacts with the output to present the processed data is known as the output layer. Layers between the input and the output layer that do not have any interaction with the environment are known as hidden layers. Increasing the complexity of an $\mathrm{ANN}$, and thus its computational capacity, re- quires the addition of more hidden layers, and more neurons per layer (Wikibooks, 2011). Processed cheese is one of the most popular varieties among the types of cheeses. Processed cheese is prepared by using medium ripened (up to 6 months old) grated Cheddar cheese by adding water, emulsifiers, salt, and preservatives by heating to $70^{\circ} \mathrm{C}$ for $10-15$ minutes with steam in a cleaned double jacketed stainless steel kettle (which is open, shallow and round-bottomed) with continuous gentle stirring (about 50-60 circular motions per minute) with a flattened ladle in order to get unique body \& texture in the product.

Linear layers are single layers of linear neurons. They may be static, with input delays of 0 , or dynamic, with input delays greater than 0 . They can be trained on simple linear time series problems, but often are used adaptively to continue learning while deployed, so they can adjust to changes in the relationship between inputs and outputs while being used (Mathworks Website 1, 2011).

Generalized regression neural network models are a kind of radial basis network that is used for function approximation. 
Syntax: net $=$ newgrnn $(P, T$, spread $)$

net $=$ newgrnn $(P, T$, spread $)$ takes three inputs:

P: R-by-Q matrix of Q input vectors;

T: S-by-Q matrix of $Q$ target class vectors.

Spread: Spread of radial basis functions (default $=1.0$ ) and returns is a new generalized regression neural network. The larger the spread, the smoother is the function approximation. To fit data very closely, a smaller spread is used than the typical distance between input vectors. To fit the data more smoothly, a larger spread is used. Newgrnn creates a two-layer network. The first layer has radbas neurons, and calculates weighted inputs with dist and net input with netprod. The second layer has purelin neurons, calculates weighted input with normprod, and net inputs with netsum. Only the first layer has biases; newgrnn sets, the first layer weights to $\mathrm{P}^{\prime}$, and the first layer biases are all set to $0.8326 /$ spread, resulting in radial basis functions that cross 0.5 at weighted inputs of $+/-$ spread. The second layer weights W2 are set to T (Mathworks Website 2, 2011).

Shelf life is defined as the length of time that a product is acceptable and meets the consumer's expectations regarding food quality. It is the result of the conjunction of all services in production, distribution, and consumption. Shelf life dating is one of the most difficult tasks in food engineering. The food engineers are facing the challenges to monitor, diagnose, and control the quality and safety of food products. Nanotechnology, multivariate sensors, information systems, and complex systems have revolutionised the food industry. The consumer demands foods under the legal standards, at low cost, high standards of nutritional, sensory, and health benefits (Martins et al., 2008).

Goyal and Goyal (2011a) implemented brain based artificially intelligent scientific computing models for shelf life detection of cakes stored at $30 \mathrm{oC}$. The potential of simulated neural networks for predicting shelf life of soft cakes stored at $10 \mathrm{oC}$ was highlighted by Goyal and Goyal (2011b). Cascade single and double hidden layer models were developed and compared with each other for predicting the shelf life of Kalakand (Goyal and Goyal, 2011c). For forecasting the shelf life of instant coffee drink, radial basis artificial neural engineering and multiple linear regression models were developed (Goyal and Goyal, 2011d). Cascade forward and feedforward backpropagation artificial intelligence models for prediction of sensory quality of instant coffee flavoured sterilized drink have been evolved (Goyal and Goyal, 2011e). Artificial neural networks for predicting the shelf life of milky white dessert jeweled with pistachio were applied by Goyal and Goyal (2011g). The shelf life of brown milk cakes decorated with almonds was predicted by developing artificial neural network based radial basis (exact fit) and radial basis (fewer neurons) models, and the developed models were compared with each other (Goyal and Goyal, 2011h).Also, the time-delay and linear layer (design) intelligent computing expert system models have been developed for predicting shelf life of soft mouth melting milk cakes stored at $60 \mathrm{C}$ (Goyal and Goyal, 2011i).

\section{METHOD MATERIAL}

The experimental data pertaining to soluble nitrogen, $\mathrm{pH}$, standard plate count, Yeast \& mould count, and spore count were used as input variables; and sensory score was taken as output variable for developing computational intelligence models (Figure 1). Experimentally obtained 36 observations for each input and output variables were taken for development of the models. The dataset was randomly divided into two disjoint subsets, namely, training set having $30(80 \%$ for training) observations, and validation set ( $20 \%$ for testing) consisting of 6 observations (Figure 2).

Prediction Performance Measures:

$$
\begin{aligned}
& M S E=\left[\sum_{1}^{N}\left(\frac{Q_{\exp }-Q_{c a l}}{n}\right)^{2}\right] \\
& R M S E=\sqrt{\frac{1}{n}\left[\sum_{1}^{N}\left(\frac{Q_{\exp }-Q_{c a l}}{Q_{\exp }}\right)^{2}\right]}
\end{aligned}
$$




$$
\begin{aligned}
& R^{2}=1-\left[\sum_{1}^{N}\left(\frac{Q_{\text {exp }}-Q_{\text {cal }}}{Q_{\exp }^{2}}\right)^{2}\right] \\
& E^{2}=1-\left[\sum_{1}^{N}\left(\frac{Q_{\text {exp }}-Q_{\text {cal }}}{Q_{\text {exp }}-\overline{Q_{\text {exp }}}}\right)^{2}\right]
\end{aligned}
$$

Where,

$Q_{\exp }=$ Observed value;

$Q_{\text {cal }}=$ Predicted value;

$\bar{Q}_{\text {exp }}=$ Mean predicted value;

$n=$ Number of observations in dataset.

Soluble nitrogen

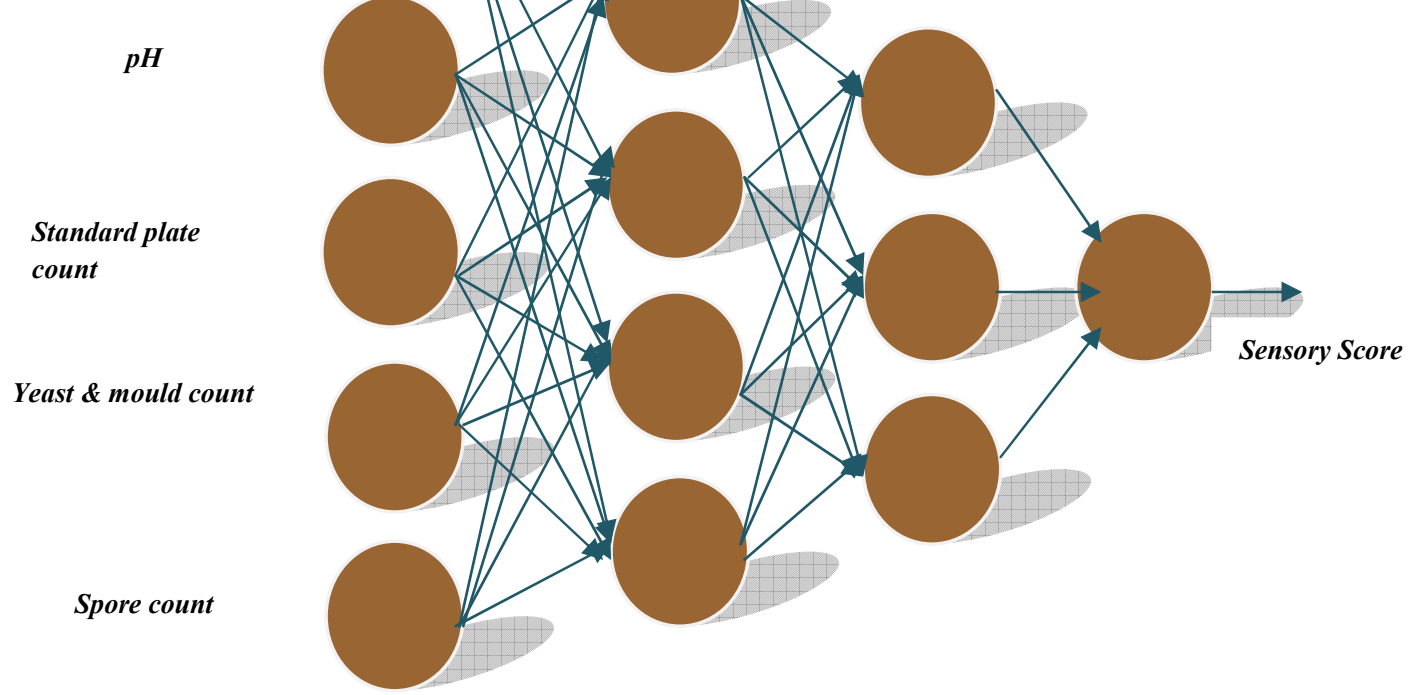

Figure 1 - Input and output parameters for computational intelligence models

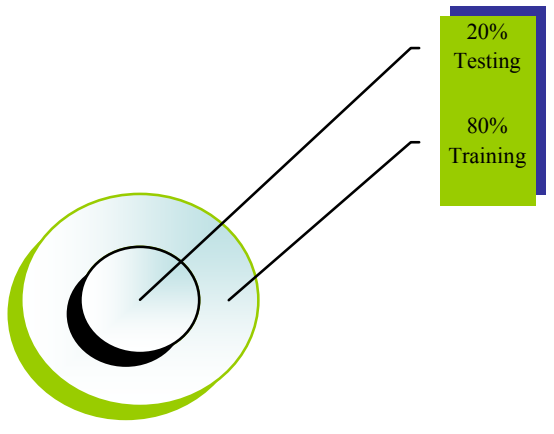

Figure 2 - Data Division

Mean Square Error MSE (1), Root Mean Square Error RMSE (2), Coefficient of Determination $R^{2}(3)$ and Nash - Sutcliffo Coefficient $E^{2}$
(4) were applied in order to compare the prediction ability of the developed models.

The best training procedure is to compile a wide range of examples (for more complex problems, more examples are required), which exhibit all the different characteristics of the problem. To create a robust and reliable network, in some cases, some noise or other randomness is added to training data to get the network familiarized with noise and natural variability in real data. Poor training data inevitably leads to an unreliable and unpredictable network. Usually, the network is trained for a prefixed number of epochs or when the output error decreases below a particular error threshold. Special care is taken not to over train the network. By overtraining, the network may become too adapted in learning the samples from the training set, and thus may be unable to accurately classify samples outside of the training set (Softcomputing Website, 2011). 


\section{RESULTS AND DISCUSSION}

Linear Layer (Table 1) and Generalized Regression models (Table 2) were developed and compared with each other for predicting shelf life of processed cheese.

The comparison of Actual Sensory Score (ASS) and Predicted Sensory Score (PSS) for the computational intelligent models are illustrated in Figure 3 and Figure 4, respectively.

On comparing the two developed models, viz., Linear Layer (MSE: 0.000115628, RMSE:
0.010753058, R2: $0.989246942, \quad$ E2: 0.999884372 ), and Generalized Regression (MSE: 1.22701E-05, RMSE : 0.003502871,R2: $0.996497129, \mathrm{E} 2: 0.99998773)$ with 10 as spread constant, it was observed that Generalized Regression model with spread constant as 10 got simulated best with less than 1\% RMSE after supervised training of ANN's. The investigation suggested that the computational intelligence models are quite efficient in predicting the shelf life of processed cheese stored at $7-8^{\circ} \mathrm{C}$.

Table 1 - Results of Linear Layer model

\begin{tabular}{|c|c|c|c|}
\hline$M S E$ & $R M S E$ & $R^{2}$ & $E^{2}$ \\
\hline 0.000115628 & 0.010753058 & 0.989246942 & 0.999884372 \\
\hline
\end{tabular}

Table 2 - Results of Generalized Regression model

\begin{tabular}{|c|c|c|c|c|}
\hline Spread Constant & MSE & RMSE & $\mathbf{R}^{2}$ & $\mathbf{E}^{2-}$ \\
\hline 10 & $1.22701 \mathrm{E}-05$ & 0.003502871 & 0.996497129 & 0.99998773 \\
\hline 20 & $2.41732 \mathrm{E}-05$ & 0.00491662 & 0.99508338 & 0.999975827 \\
\hline 30 & $6.72027 \mathrm{E}-05$ & 0.008197726 & 0.991802274 & 0.999932797 \\
\hline 40 & $8.01244 \mathrm{E}-05$ & 0.008951222 & 0.991048778 & 0.999919876 \\
\hline 50 & 0.022592593 & 0.150308325 & 0.849691675 & 0.977407407 \\
\hline 60 & $8.74111 \mathrm{E}-05$ & 0.00934939 & 0.99065061 & 0.999912589 \\
\hline 70 & $8.87355 \mathrm{E}-05$ & 0.009419951 & 0.990580049 & 0.999911265 \\
\hline 80 & $8.94967 \mathrm{E}-05$ & 0.009460272 & 0.990539728 & 0.999910503 \\
\hline 90 & $9.0022 \mathrm{E}-05$ & 0.009487993 & 0.990512007 & 0.999909978 \\
\hline 100 & $9.03571 \mathrm{E}-05$ & 0.009505633 & 0.990494367 & 0.999909643 \\
\hline 110 & $9.05968 \mathrm{E}-05$ & 0.009518233 & 0.990481767 & 0.999909403 \\
\hline 120 & $9.07888 \mathrm{E}-05$ & 0.009528313 & 0.990471687 & 0.999909211 \\
\hline 130 & $9.09329 \mathrm{E}-05$ & 0.009535874 & 0.990464126 & 0.999909067 \\
\hline 140 & $9.10771 \mathrm{E}-05$ & 0.009543434 & 0.990456566 & 0.999908923 \\
\hline 150 & $9.11252 \mathrm{E}-05$ & 0.009545954 & 0.990454046 & 0.999908875 \\
\hline 160 & $9.12215 \mathrm{E}-05$ & 0.009550994 & 0.990449006 & 0.999908779 \\
\hline 170 & $9.12696 \mathrm{E}-05$ & 0.009553514 & 0.990446486 & 0.99990873 \\
\hline 180 & $9.13178 \mathrm{E}-05$ & 0.009556034 & 0.990443966 & 0.999908682 \\
\hline 190 & $9.1366 \mathrm{E}-05$ & 0.009558554 & 0.990441446 & 0.999908634 \\
\hline 200 & $9.14141 \mathrm{E}-05$ & 0.009561074 & 0.990438926 & 0.999908586 \\
\hline
\end{tabular}

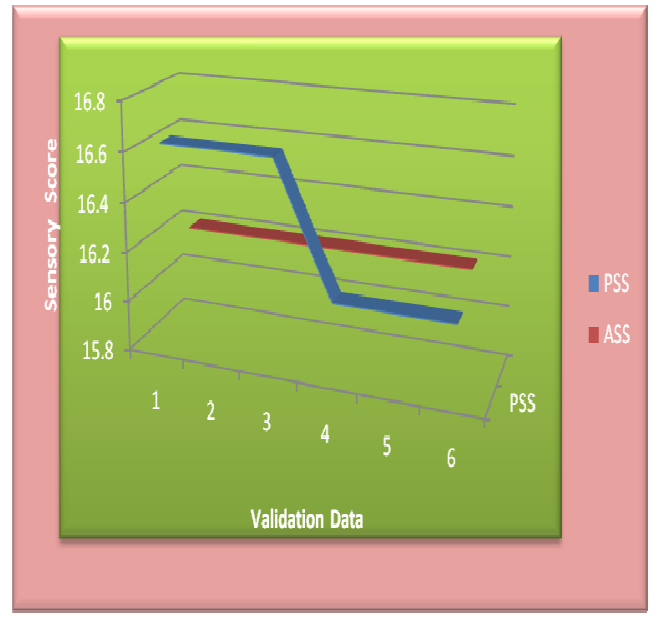

Figure 3 - Comparison of ASS and PSS for Linear Layer model

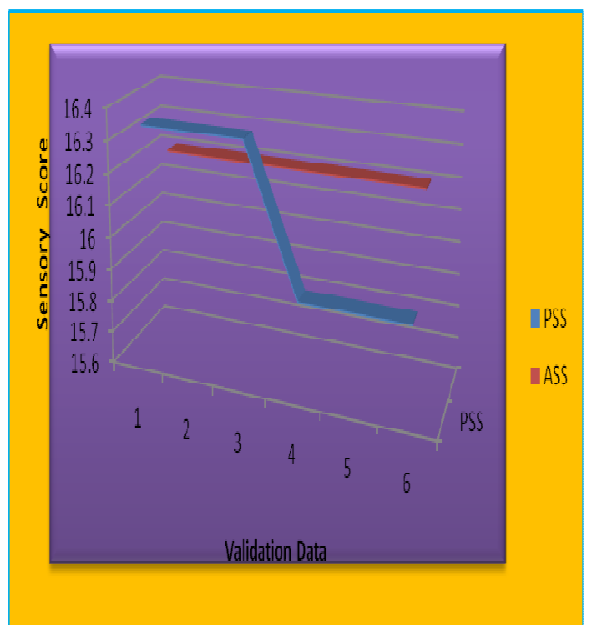

Figure 4 - Comparison of ASS and PSS for Generalized Regression model 


\section{CONCLUSION}

Linear Layer and Generalized Regression models were developed for predicting the shelf life of processed cheese stored at 7-8oC. Soluble nitrogen, $\mathrm{pH}$, Standard plate count, Yeast \& mould count, Spore count were taken as input variables, and the experimental sensory score was taken as output variable for developing computational intelligence models. The data consisted of 36 experimental observations, which were sub- divided into two sets, i.e., 30 ( $80 \%$ of observations) for training, and 6 ( $20 \%$ of observations) for validation of the developed models. The comparison of the two developed models showed that Generalized Regression model with spread constant as 10 got best simulated with less than $1 \%$ RMSE. The study led to conclusion that computational intelligence models are quite effective in predicting the shelf life of processed cheese stored at $7-8^{\circ} \mathrm{C}$.

\section{REFERENCES}

Goyal, Sumit, \& Goyal, G.K. (2011a). Brain based artificial neural network scientific computing models for shelf life prediction of cakes. Canadian Journal on Artificial Intelligence, Machine Learning and Pattern Recognition, 2(6), 73-77.

Goyal, Sumit, \& Goyal, G. K. (2011b). Simulated neural network intelligent computing models for predicting shelf life of soft cakes. Global Journal of Computer Science and Technology, 11(14), Version 1.0, 29-33.

Goyal, Sumit, \& Goyal, G.K. (2011c). Advanced computing research on cascade single and double hidden layers for detecting shelf life of kalakand: An artificial neural network approach. International Journal of Computer Science \& Emerging Technologies, 2(5), 292-295.

Goyal, Sumit, \& Goyal, G.K. (2011d). Application of artificial neural engineering and regression models for forecasting shelf life of instant coffee drink. International Journal of Computer Science Issues, 8(4), No 1, 320324.

Goyal, Sumit, \& Goyal, G.K. (2011e).Cascade and feedforward backpropagation artificial neural networks models for prediction of sensory quality of instant coffee flavoured sterilized drink. Canadian Journal on Artificial Intelligence, Machine Learning and Pattern Recognition, 2(6), 78-82.

Goyal, Sumit, \& Goyal, G.K. (2011f). Development of neuron based artificial intelligent scientific computer engineering models for estimating shelf life of instant coffee sterilized drink. International Journal of Computational Intelligence and Information Security, 2(7), 4-12.
Goyal, Sumit, \& Goyal, G.K. (2011g). A new scientific approach of intelligent artificial neural network engineering for predicting shelf life of milky white dessert jeweled with pistachio. International Journal of Scientific and Engineering Research, 2(9), 1-4.

Goyal, Sumit, \& Goyal, G.K. (2011h). Radial basis artificial neural network computer engineering approach for predicting shelf life of brown milk cakes decorated with almonds. International Journal of Latest Trends in Computing, 2(3), 434-438.

Goyal, Sumit, \& Goyal, G.K. (2011i). Development of intelligent computing expert system models for shelf life prediction of soft mouth melting milk cakes. International Journal of Computer Applications, 25(9), 41-44.

Martins, R. C., Lopes, V.V., Vicente, A.A. \& Teixeira, J.A.(2008).Computational shelflife dating: complex systems approaches to food quality and safety. Food and Bioprocess Technology, 1(3), 207-222.

Mathworks Website 1: http://www.mathworks.com/help/toolbox/nn et/ref/linearlayer.html (accessed on 15.4.2011).

Mathworks Website 2: http://www.mathworks.com/help/toolbox/nn et/ref/newgrnn.html (accessed on 1.5.2011).

Softcomputing Website: http://www.softcomputing.net/ann chapter.p df (accessed on 1.5.2011)

Wikibooks Website: http://en.wikibooks.org/wiki/Artificial_Neur al Networks/Neural Network Basics (accessed on 21.5.2011). 